\title{
Disease Control Status and Safety of Telemedicine in Patients With Lifestyle Diseases \\ - A Multicenter Prospective Observational Study in Japan -
}

\author{
Yoshito Kadoya, MD; Masahiko Hara, MD, PhD; Kosuke Takahari, MD; \\ Yoko Ishida, MD; Masatake Tamaki
}

\begin{abstract}
Background: The Japanese Ministry of Health, Labour and Welfare has tried to promote telemedicine since 2018, but faces difficulties in increasing the use of telemedicine partly due to a lack of clinical evidence. This study investigated the disease control status and safety of telemedicine, which, in Japan, is provided under the National Health Insurance system, for the treatment of lifestyle diseases under the present legal restraints.
\end{abstract}

Methods and Results: This multicenter prospective observational study started in April 2018 and enrolled 34 patients with lifestyle diseases, including hypertension, dyslipidemia, and diabetes. Primary and secondary outcome measures included control status, serial changes in clinical indices, and the safety of telemedicine 6 months after implementation. Control status was assessed by the attending physician, and differences in blood pressure (BP), low density lipoprotein cholesterol (LDL-C), or HbA1c levels were evaluated. Of the 34 patients, 29 were successfully introduced to telemedicine and followed-up for 6 months. Median patient age was 77 years, 14 (48.3\%) were men, 24 (82.8\%) had hypertension, 17 (58.6\%) had dyslipidemia, and $9(31.0 \%)$ had diabetes. At the 6-month follow-up, no patients had experienced exacerbation of underlying diseases, with no significant changes in BP, LDL-C, or HbA1c. Moreover, no telemedicine-associated adverse events were observed.

Conclusions: Telemedicine can be a safe and feasible option for managing lifestyle diseases under the present legal restraints.

Key Words: Lifestyle disease; Ministry of Health, Labour and Welfare; Telemedicine

$\mathbf{T}$ elemedicine is defined as the use of information and communication technologies (ICTs) to improve patient outcomes by providing accessible, costeffective, high-quality healthcare services. ${ }^{1}$ Telemedicine uses ICTs mainly to liberate patients and medical professionals from geographical barriers and time restrictions. The recent concept of telemedicine is extremely wide, ranging from diagnosis to therapeutics. ${ }^{2,3}$

Telemedicine includes the provision of consultations and medical care to both patients and peers by medical professionals regardless of real time through means such as video, conversation, or text chat tools, and even includes e-learning services for self-medication. Telemedicine is commonly used as a therapeutic tool for the management of lifestyle diseases, and a wide variety of evidence as to the effectiveness of telemedicine in this field is available. ${ }^{4-10}$

With the world-wide dissemination of telemedicine, as well as its clinical benefits and cost effectiveness, the Japanese
Ministry of Health, Labour and Welfare (MHLW) has made efforts to promote telemedicine since 2015, and National Health Insurance can be applied to the management of lifestyle diseases as long as the guidelines for the appropriate operation of online medicine using real-time interactive video-based tools for telemedicine released in March 2018 are followed. ${ }^{11}$ However, telemedicine has not yet been widely adopted, even after the implementation of the New Health Insurance system and guidelines for the appropriate operation of online medicine in 2018, partly because of a lack of economic advantage or because of the unique and strict regulations that vitiate the temporal and geographical merits of telemedicine, such as the requirement for face-to-face consultations with a fixed attending physician every 3 months. ${ }^{11}$ In fact, according to the MHLW, only 65 telemedicine-based episodes of medical care are performed per month throughout Japan. ${ }^{12}$ A lack of clinical evidence regarding the benefits of telemedicine under the

Received February 23, 2020; revised manuscript received April 29, 2020; accepted May 12, 2020; J-STAGE Advance Publication released online June 3, 2020 Time for primary review: 38 days

Department of Cardiovascular Medicine, Graduate School of Medical Science, Kyoto Prefectural University of Medicine, Kyoto (Y.K.); Center for Community-Based Healthcare Research and Education, Shimane University Graduate School of Medicine, Izumo (M.H.); Department of Clinical Investigation, Japan Society of Clinical Research, Osaka (M.H., M.T.); Shobara City Soryo National Health Insurance Clinic, Hiroshima (K.T.); and Department of Occupational and Environmental Health, Shinyo Clinic, Tokyo (Y.I.), Japan

Mailing address: Masahiko Hara, MD, PhD, Center for Community-Based Healthcare Research and Education, Shimane University Graduate School of Medicine, 89-1 Enyacho, Izumo 693-8501, Japan. E-mail: hara@japanscr.org

All rights are reserved to the Japanese Circulation Society. For permissions, please e-mail: cr@j-circ.or.jp

ISSN-2434-0790 
current regulations may also hamper its spread throughout Japan.

Accordingly, the aim of this study was to investigate disease control status, serial changes in clinical indices, and the safety of video-based interactive telemedicine under the current Japanese regulations for the treatment of lifestyle diseases. Our aim is to contribute to catching up to world standards for the provision of telemedicine in Japan by collecting evidence, revealing any problems associated with the unique regulations that vitiate the merits of telemedicine, and deregulating the unique, strict restrictions regarding telemedicine in Japan.

\section{Methods}

\section{Study Population}

This study was designed as a multicenter prospective observational study that enrolled patients with lifestyle diseases, such as hypertension, dyslipidemia, and diabetes, who received telemedicine consultations under the regulations of the National Health Insurance system in Japan between April 2018 and October 2019. The definitions for each lifestyle disease were based on the guidelines at the time of study design. Specifically, hypertension was defined according to the Japanese Society of Hypertension Guidelines as systolic/diastolic blood pressure (SBP/DBP) $\geq 140 / 90 \mathrm{mmHg}$ in an outpatient setting, home SBP/DBP $\geq 135 / 85 \mathrm{mmHg}$, or the use of antihypertensive medications. ${ }^{13}$ Dyslipidemia was defined according to the Japan Atherosclerosis Society Guidelines as total cholesterol (TC) $\geq 220 \mathrm{mg} / \mathrm{dL}$, low-density lipoprotein cholesterol (LDL-C) $\geq 140 \mathrm{mg} / \mathrm{dL}$, high-density lipoprotein cholesterol (HDL-C) $<40 \mathrm{mg} / \mathrm{dL}$, triglycerides (TG) $\geq 150 \mathrm{mg} / \mathrm{dL}$, or the use of medications to control one of these parameters. ${ }^{14}$ Diabetes was defined on the basis of Japan Diabetes Society guidelines as fasting blood glucose (FBG) $\geq 126 \mathrm{mg} / \mathrm{dL}$, 2-h blood glucose after an oral glucose tolerance test $\geq 200 \mathrm{mg} / \mathrm{dL}$, $\mathrm{HbA} 1 \mathrm{c} \geq 6.5 \%$ met twice in each combination, or the use of any antidiabetic medication, including insulin. ${ }^{15,16}$

All patients included in the study were determined to be appropriate candidates for telemedicine by their attending physician and were scheduled to participate in telemedicine consultations under the National Health Insurance system in Japan. Exclusion criteria were set on the basis of the definition of appropriate candidates for telemedicine by the MHLW, and included: patients with severe cardiac, liver, or renal diseases; patients with malignancy under treatment; participants within 3 months of other clinical studies; patients unable to understand the study protocol due to psychiatric disorder, etc.; and other participants considered inappropriate by their attending physician.

The study protocol complied with the Declaration of Helsinki and was approved by the ethics committee of each participating hospital or clinic after it had gained approval from the Institutional Review Board (IRB) of the Japan Society of Clinical Research (IRB ID 17000059). All patients provided written informed consent prior to taking part in the study. This trial was registered with the University Hospital Medical Information Network Clinical Trials Registry, as accepted by the International Committee of Medical Journal Editors (UMIN-ID; 000032643).

\section{Provision of Telemedicine and Data Capture}

Patients received real-time interactive video-based telemedicine via a smartphone or tablet device following the regulations of the National Health Insurance system in Japan. The Japanese insurance system require patients to have face-to-face consultations at least every 3 months with their attending physician. In addition, telemedicine must be provided by an attending physician who has provided a face-to-face consultations to the patient every month for 6 months or at least 6 consultations within a 12-month period at the time of telemedicine consultation. Thus, mandates of real-time interactive communication and face-to-face consultations at least every 3 months vitiate the most important merits of telemedicine, namely freedom from time restrictions and geographical barriers. Failure to follow these regulations incurs a risk of violating the Article 20 of the Japanese Medical Practitioners Law.

The scheduling of telemedicine and face-to-face visits was at the discretion of the attending physician. However, the study protocol recommended an office visit every 3 months ( 3 and 6 months after study enrollment) with complementary telemedicine consultations each month between office visits (i.e., 1, 2, 4, and 5 months after study enrollment). Video communication applications were handled by the patients, their family members, or by caregivers, including home visit nurses. Payments were made using web-based payment tools, bank deposits, or as lump sum cash payments during face-to-face visits. Prescriptions could be delivered by mail or in person at face-to-face visits, as preferred by the patient. All information shown in the tables was prespecified and recorded prospectively on an electrical data-capture system.

\section{Endpoints and Statistical Analysis}

The primary endpoint was the control status of the lifestyle disease 6 months after the implementation of telemedicine. The control status of each lifestyle disease was divided into 3 categories: improved, no change, or exacerbated (coded as $-1,0$, and +1 , respectively). Control status was assessed clinically by the attending physicians. If patients had multiple lifestyle diseases, the sum of their respective codes served as an index of the comprehensive control status of the patient. Secondary endpoints included the control status of each underlying lifestyle disease individually, serial changes in disease-related indices (e.g., SBP/DBP in an outpatient setting for hypertension; laboratory data for TC, LDL-C, HDL-C, and TG for dyslipidemia; HbAlc and FBG for diabetes), and the number of drugs prescribed for each underlying lifestyle disease. The incidence of telemedicine-related and any other types of adverse events was also evaluated as a secondary endpoint in the entire study population. In addition, patient satisfaction was assessed using a questionnaire.

Continuous variables are presented as the median and interquartile range, whereas categorical variables are presented as counts and percentages. The significance of differences in clinical indices between baseline and the 6-month follow-up after implementation of telemedicine was analyzed using the Wilcoxon signed-rank test considering parity to maintain statistical power in this study with a small sample size. For example, the statistical power was 0.95 with a sample size of 15 participants if we set the difference, standard deviation, and $\alpha$ error of the variables as 10,10 , and 0.05 , respectively. This is applicable for all paired comparisons of continuous variables, namely some of the secondary endpoints, such as SBP/DBP, TC, LDL-C, HDL-C, TG, HbAlc, and FBG levels. Statistical analyses were performed using Microsoft R Open version 3.3.2 


\begin{tabular}{|lc|}
$\begin{array}{l}\text { Table 1. Baseline Characteristics of the Study Population } \\
(\mathbf{n}=\mathbf{2 9})\end{array}$ & $77[68-84]$ \\
Age (years) & $14(48.3)$ \\
Male sex & $24.1[20.1-26.2]$ \\
Body mass index $\left(\mathbf{k g} / \mathbf{m}^{2}\right)$ & $6(20.7)$ \\
Patients with a job & $3(10.3)$ \\
Part-time & $3(10.3)$ \\
Full-time & $3(10.3)$ \\
Current smoker & \\
Baseline diseases and medications used & $24(82.8)$ \\
Hypertension & $19(65.5)$ \\
CCBs & $13(44.8)$ \\
ACEIs/ARBs & $17(58.6)$ \\
Dyslipidemia & $15(51.7)$ \\
Statins & $2(6.9)$ \\
Fibrates & $9(31.0)$ \\
Diabetes & $5(17.2)$ \\
DPP4ls & $4(13.8)$ \\
Biguanides & $1(3.4)$ \\
SGLT2i & $1(3.4)$ \\
aGls & $2(6.9)$ \\
Insulin & \\
\hline
\end{tabular}

Continuous variables are presented as the median [interquartile range] and categorical data are presented as n (\%). ACEI, angiotensin-converting enzyme inhibitors; ARBs, angiotensin receptor blockers; CCBs, calcium channel blockers; DPP4Is, dipeptidyl peptidase 4 inhibitors; $a \mathrm{Gls}$, $a$-glucosidase inhibitors; SGLT2i, sodium-glucose cotransporter inhibitors.

(Microsoft Corp., Bellevue, WA, USA). The significance level for statistical hypothesis testing was set at 0.05 and the alternative hypothesis was 2 -sided.

\section{Results}

Among the 34 registered patients who provided written informed consent, 2 were excluded after dropping out of the study because of unfamiliarity with smartphones or tablet devices and another 3 were excluded because they did not receive any telemedicine consultations during the study period in part because they saw no merits in it (e.g., freedom from time restrictions), despite their agreement to try telemedicine at the time of enrollment. This left 29 patients who were successfully followed up for 6 months and analyzed as the final study population. There were no missing data regarding primary and secondary endpoints in this study.

Baseline patient characteristics are given in Table 1. The median age of the study population was 77 years, and 14 $(48.3 \%)$ patients were male. Six $(20.7 \%)$ patients were employed, 24 (82.8\%) had hypertension, 17 (58.6\%) had dyslipidemia, and $9(31.0 \%)$ had diabetes. Telemedicinerelated information is summarized in Table 2 . In the case of 14 patients $(48.2 \%)$, telemedicine was started in order to save the time of hospital visits, whereas for $13(44.8 \%)$ telemedicine was started to reduce the burden of family attendance associated with the patient's hospital visits. Fourteen $(48.3 \%)$ patients were able to operate the smartphone or tablet device on their own, whereas approximately half the study population needed the support of a family member, home visit nurse, or nursing home staff. In most cases, payment was made when visiting the hospital for

\begin{tabular}{|lc|}
\hline $\begin{array}{l}\text { Table 2. Telemedicine-Related Information for the Study } \\
\text { Population ( } \mathbf{n}=29)\end{array}$ & $\begin{array}{c}\text { No. patients } \\
(\%)\end{array}$ \\
Reason for introducing telemedicine & $14(48.2)$ \\
To save time of hospital visits & $13(44.8)$ \\
$\quad \begin{array}{l}\text { To reduce the burden on family attending } \\
\text { hospital visits }\end{array}$ & $2(6.9)$ \\
Other & \\
Device handling & $14(48.3)$ \\
By the patient himself/herself & $10(34.5)$ \\
By the patient's family & $4(13.8)$ \\
By a home visit nurse & $1(3.4)$ \\
By nursing home staff & \\
Payment method & $28(96.6)$ \\
Cash at the face-to-face consultation & $1(3.4)$ \\
\hline Bank deposit & $0(0.0)$ \\
Online payment & $24(82.8)$ \\
Method of delivering prescriptions to patients & $4(13.8)$ \\
In person at the hospital or clinic & $1(3.4)$ \\
\hline In person at the pharmacy & \\
By mail &
\end{tabular}

face-to-face medical consultations, and prescriptions were handed over at the hospital at the time of the face-to-face visit.

The results regarding primary and secondary endpoints are given in Table 3. Generally, the control status of lifestyle diseases remained unchanged in most of the study population as per the recent regulations in Japan, with 26 $(89.7 \%)$ patients showing no change and remaining 3 $(10.3 \%)$ patients showing an improvement in control status during the 6-month study period. In addition, there were no telemedicine-related adverse events, although 2 patients (72- and 93-year-old males) were hospitalized for the management of bacterial pneumonia during the study period. Most patients in the present study $(88.0 \%)$ were satisfied with no waiting time for consultations and had sufficient time to talk to the doctor.

In subgroup analyses, there were no significant changes in disease-specific continuous indices, such as SBP and DBP in patients with hypertension $(n=24), T C$, LDL-C, HDL-C, and TG in patients with dyslipidemia $(n=17)$, or HbAlc and FBG in patients with diabetes $(n=9)$. With regard to the control status of each underlying lifestyle disease, 4 patients with hypertension were considered to have improved, whereas 3 received additional prescriptions of antihypertensive drugs. No specific changes were observed in the control status or the number of medications among patients with dyslipidemia. In contrast, 2 patients with diabetes were able to decrease the number of medications taken without any clinical changes in the control status of their condition.

\section{Discussion}

In this study we investigated disease control status, serial changes in clinical indices, and the safety of real-time interactive telemedicine for the treatment of lifestyle diseases. Even after the implementation of telemedicine, the control status of lifestyle disease remained unchanged in most of 
Table 3. Outcome Data

Total study population $(\mathbf{n}=\mathbf{2 9})$

Comprehensive disease control status of the patient

Improved

No change

$26(89.7)$

Exacerbated

$0(0.0)$

Telemedicine-related adverse events

Any kind of adverse event ${ }^{A}$

$2(6.9)$

Follow-up periods (days)

$196[168-210]$

No. consultations over 6 months

$\begin{array}{ll}\text { Face-to-face } & 3[2-3] \\ \text { Telemedicine } & 2[2-3] \\ \text { Patient satisfaction } & 9[8-10]\end{array}$

Reason for satisfaction with telemedicine ${ }^{B}$

No waiting time for consultation $\quad 22(88.0)$

No need to visit the hospital $\quad 16(64.0)$

Low cost of consultation $\quad 17(68.0)$

Sufficient time to talk to the doctor $\quad 22(88.0)$

Patients with hypertension $(n=24)$

Baseline

At 6 months

P-value

Disease control status

Improved

No change

Exacerbated

SBP $(\mathrm{mmHg})$

DBP $(\mathrm{mmHg})$

No. medications

Increased

Unchanged

Decreased

- $\quad 4(16.7)$

- $\quad 20(83.3)$

- $\quad 0(0.0)$

$0(0.0)$

$138[123-148]$

75 [67-83]

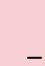

atients with dyslipidemia $(n=17)$

Disease control status

Improved

No change

Exacerbated

$\mathrm{TC}(\mathrm{mg} / \mathrm{dL})$

LDL-C (mg/dL)

71 [64-80]

3 (12.5)

$\begin{array}{rr}- & 3(12.5) \\ - & 19(79.2)\end{array}$

- $2(8.3)$

HDL-C (mg/dL)

Triglycerides (mg/dL)

No. medications

Increased

Unchanged

Decreased

tients with diabetes $(n=9)$

Disease control status

Improved

No change

Exacerbated

$\mathrm{HbA1c}(\%)$

Blood glucose (mg/dL)

No. medications
Increased
Unchanged
Decreased

-
-
-
$158[144-186]$
$81[70-93]$
$52[44-70]$
$162[93-208]$

$0(0.0)$
$17(100.0)$
$0(0.0)$

$158[145-198]$

87 [73-105]

50 [44-75]

130 [87-207]

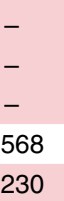

0.230

0.653

Categorical variables are presented as $n(\%)$ and continuous variables are presented as the median [interquartile range]. The significance of differences in continuous variables between baseline and 6 months after the implementation of telemedicine was determined using the Wilcoxon rank-sum test. AHospitalization due to bacterial pneumonia. ${ }^{\mathrm{B} P a t i e n t}$ satisfaction with telemedicine was evaluated using a questionnaire. The questionnaire was completed by 25 patients $(86.2 \%)$, with satisfaction scored from 1 to 10 , with 1 being completely unsatisfied and 10 being completely satisfied. DBP, diastolic blood pressure; HDL-C, high-density lipoprotein cholesterol; LDL-C, low-density lipoprotein cholesterol; SBP, systolic blood pressure; TC, total cholesterol. 
the study population. Moreover, no telemedicine-related adverse events were observed. We demonstrated that telemedicine can be a safe and feasible option in controlling lifestyle diseases under current regulations in Japan. Because there is a significant lack of evidence regarding telemedicine under the latest regulations in Japan, this study provides physicians with new insights into this field.

\section{Utility of Telemedicine in Japan}

We demonstrated that telemedicine can be a safe and feasible option for controlling lifestyle diseases under the current National Health Insurance system and guidelines for the appropriate operation of online medicine, promulgated in March 2018 by the MHLW.

In fact, many studies have been published showing the effectiveness of telemedicine in the treatment of various chronic diseases, such as hypertension, dyslipidemia, and diabetes, in other countries. ${ }^{3-10}$ For example, Artinian et al reported a greater decrease in mean SBP in telemedicine delivered in addition to face-to-face consultations than in face-to-face consultations alone at 12 months. ${ }^{4}$ Davis et al showed that lower LDL-C levels were achieved using telemedicine in patients with diabetes, ${ }^{7}$ and Stone et al demonstrated significantly greater reductions in $\mathrm{HbAlc}$ by 3 and 6 months in the home telemonitoring group than with usual care. ${ }^{8}$ The results of the present study are consistent with these findings from overseas.

Although physical safety risks associated with telemedicine can be common in daily clinical practice, ${ }^{1-3}$ we think that the possible loss of opportunities from the unexpected detection of signs of illness due to a lack of physical examination could be a more concrete issue pertaining to the long-term provision of telemedicine. However, we speculate that this problem could be avoided if telemedicine is combined with periodic face-to-face consultations, as recommended by the MHLW.

We believe that the problem of a small sample size $(n=29)$ in this study has only little importance in clinical decision making because the study design used paired comparisons of continuous variables, such as blood pressure, cholesterol, or $\mathrm{HbAlc}$, as secondary endpoints in order to maintain the statistical validity of the results, as described in the Methods. In addition, this number could be one of the largest reported, considering that, according to the MHLW, only 65 telemedicine-based episodes of medical care are performed per month throughout Japan. ${ }^{12}$ For these reasons, we think that our data can provide helpful evidence for physicians in this field.

\section{Issues of Telemedicine in Japan}

Unfortunately, 5 patients were excluded from the final analysis. Two patients dropped out because of unfamiliarity with smartphone or tablet devices. A survey by the Ministry of Internal Affairs and Communications in 2017 found that ownership of ICT equipment decreases with age, with smartphone ownership only $8.4 \%$ in those $>75$ years of age. ${ }^{17}$ In the present study, the median age of the sample was 77 years, and fewer than half the participants could handle their devices for telemedicine by themselves. Support from family members or nursing home staff was helpful for $11(37.9 \%)$ patients. Interestingly, we also demonstrated that the combination of home visit nursing care and telemedicine was effective for $4(13.8 \%)$ patients. Because support from a home visit nurse could provide several benefits, such as smooth device operation, the collection of clinical information (including vital signs) by nurses, and possible online doctor-to-nurse instructions, we think that a combination of home visit nursing care and telemedicine could be a useful tool for the provision of medical care in Japan. From this viewpoint, a user-friendly device that is easy to use even by patients with low digital literacy is helpful, and a system that allows medical personnel to remotely control the equipment could be an option.

In addition, 3 patients did not receive telemedicine consultations during the study period, partly because they found no merits in it, such as freedom from time restrictions, despite their agreement at the time of enrollment. As mentioned above, telemedicine uses ICTs primarily to overcome geographical barriers and time restrictions. However, recent regulations in Japan vitiate these important merits of telemedicine by requiring "real-time video" communication and forcing doctors to be located in a hospital while patients basically should be in their own house; in contrast, in other countries text-based non-real-time medical care or consultations can be performed. ${ }^{1}$ We believe that delegating the authority to make decisions as to the manner in which telemedicine is to be practiced to doctors is the crucial factor underlying the merits of telemedicine, as in other countries. We hope that the potential risk of breaching Article 20 of the Japanese Medical Practitioners Law will be remedied by the MHLW in the near future. Finally, security-related fears and the need of some patients to be seen directly by doctors could also explain why the use of telemedicine is not widespread in Japan. With regard to potential security problems, hospitals or clinics should acquire permission from the regional bureau of the MHLW to provide telemedicine services. In addition, doctors or medical staff involved in telemedicine should carefully clarify issues to patients, to abolish imaginary fears, if necessary. Nevertheless, we should remind stakeholders that telemedicine is just one of the tools available to practice medicine, and that nobody should be coerced to use telemedicine against their will. Telemedicine would not be an appropriate choice for patients who hope to receive face-to-face consultations. Therefore, it should be introduced based on patient preferences.

\section{Clinical Implications and Future Perspectives}

In this study we showed that telemedicine is a safe and feasible option for the treatment of lifestyle diseases in the current clinical setting in Japan. We hope that these data will contribute to an improvement in daily clinical practice, as well as to the resolution of social problems in Japan. At present, Japan faces many social and governmental problems, including a rapid escalation of nationwide medical costs, preventable disease onset without daily care despite prior notification of lifestyle diseases indicated by annual health check-ups, inappropriate hospital visits to the emergency department late at night requiring medication for lifestyle diseases, and a lack of human resources due to the retirement of medical doctors in their early career after delivery because there is no option for them to work in their own homes. These problems interact with each other, and we believe that telemedicine can be a strong avenue to resolve these issues if the MHLW moves to world standards by easing the recent strict regulations regarding telemedicine.

Approximately one-third of the Japanese population reportedly has a lifestyle disease, ${ }^{18}$ and most of the patients with lifestyle diseases who work remain untreated, presumably because they do not have sufficient time to visit 
hospitals during the day. If we could provide these patients with telemedicine in a non-real-time text-based manner, as in other countries, this could lead to a decrease in both inappropriate hospital visits late at night and preventable disease onset, resulting in a reduction in nationwide medical costs. Licensed medical doctors obliged to retire can work and contribute to advances in public health by working in telemedicine from their own home. The provision of telemedicine as a private practice at one's own expense may slow or even halt the rapid escalation of nationwide medical costs. We think cardiologists have a social responsibility to achieve these goals.

\section{Study Limitations}

The present study has certain limitations. First, the study included a small number of patients, which may be insufficient to fully reveal the effects and safety of telemedicine. However, it is possible that this study included a large proportion of all patients in Japan receiving telemedicine during the study period because the MHLW suggests that only 65 telemedicine-based episodes of medical care are performed each month after the regulatory notice was issued on March 2018. To overcome this limitation, we also used intragroup paired tests for statistical hypothesis testing rather than setting a control group. Second, the 6-month follow-up period of the present study was relatively short compared with the 12-month periods used in previous studies in other countries. ${ }^{3-10} \mathrm{~A}$ longer follow-up period is necessary to confirm the effects of telemedicine on lifestyle diseases. Third, the majority of the study population was elderly, whereas appropriate candidates for telemedicine include middle-aged patients with lifestyle diseases who do not receive any medications, as discussed above. Fourth, we did not evaluate the disease control status and safety of telemedicine in moderate to severe situations because present regulations limit the provision of telemedicine to cases of well-controlled diseases. Fifth, data on consultation time were not available. Finally, there was a significant selection bias in the sampling because participants were familiar with or had access to telemedicine. Such older patients may not be representative of the general population. ${ }^{17}$ These limitations should be kept in mind when interpreting the study results.

\section{Conclusions}

In conclusion, telemedicine can be a safe and feasible option for managing lifestyle diseases under the present legal restrictions in Japan.

\section{Acknowledgment}

The authors thank the Japan Society of Clinical Research for their dedicated support.

\section{Sources of Funding}

This study was funded by Mitsui \& Co., Ltd.

\section{Disclosures}

None of the authors has any financial interests to disclose or conflicts of interest to declare.

\section{IRB Information}

The study protocol was approved by the Institutional Review Board of the Japan Society of Clinical Research (IRB ID 17000059). This trial was registered with the University Hospital Medical Information Network Clinical Trials Registry, as accepted by the International Committee of Medical Journal Editors (UMIN-ID 000032643).

\section{Data Availability}

The deidentified participant data will be shared upon request to the corresponding author, and will be available for up to 3 years after the publication of this paper.

\section{References}

1. World Health Organization. Telemedicine: Opportunities and developments in member states: Report on the second global survey on eHealth 2009. Global Observatory for eHealth Series - Volume 2. https://www.who.int/goe/publications/goe_telemedicine_2010. pdf (accessed January 6, 2020).

2. Tuckson RV, Edmunds M, Hodgkins ML. Telehealth. $N$ Engl $J$ Med 2017; 377: 1585-1592.

3. Flodgren G, Rachas A, Farmer AJ, Inzitari M, Shepperd S Interactive telemedicine: Effects on professional practice and health care outcomes. Cochrane Database Syst Rev 2015; 9: CD002098.

4. Artinian NT, Flack JM, Nordstrom CK, Hockman EM, Washington OGM, Jen KL, et al. Effects of nurse-managed telemonitoring on blood pressure at 12-month follow-up among urban African Americans. Nurs Res 2007; 56: 312-322.

5. Parati G, Omboni S, Albini F, Piantoni L, Giuliano A, Revera M, et al. Home blood pressure telemonitoring improves hypertension control in general practice: The TeleBPCare study. J Hypertens 2009; 27: 198-203.

6. Bove AA, Homko CJ, Santamore WP, Kashem M, Kerper M, Elliott DJ. Managing hypertension in urban underserved subjects using telemedicine: A clinical trial. Am Heart J 2013; 165: 615-621.

7. Davis RM, Hitch AD, Salaam MM, Herman WH, ZimmerGaller IE, Mayer-Davis EJ. TeleHealth improves diabetes selfmanagement in an underserved community. Diabetes Care 2010; 33: $1712-1717$.

8. Stone RA, Rao RH, Sevick MA, Cheng C, Hough LJ, Macpherson DS, et al. Active care management supported by home telemonitoring in veterans with type 2 diabetes: The DiaTel randomized controlled trial. Diabetes Care 2010; 33: 478-484.

9. Shea S, Weinstock RS, Starren J, Teresi J, Palmas W, Field L, et al. A randomized trial comparing telemedicine case management with usual care in older, ethnically diverse, medically underserved patients with diabetes mellitus. J Am Med Inform Assoc 2006; 13: $40-51$.

10. McMahon GT, Gomes HE, Hickson Hohne S, Hu TM, Levine BA, Conlin PR. Web-based care management in patients with poorly controlled diabetes. Diabetes Care 2005; 28: 1624-1629.

11. Ministry of Health, Labour and Welfare. The guidelines for appropriate operation of online medicine [in Japanese]. https:// www.mhlw.go.jp/content/000534254.pdf (accessed January 6, 2020).

12. Ministry of Health, Labour and Welfare. Summary reports for utilization status of the health insurance system after the 2018 updates [in Japanese]. https://www.mhlw.go.jp/content/12404000/ 000547022.pdf (accessed January 6, 2020).

13. Shimamoto K, Ando K, Fujita T, Hasebe N, Higaki J, Horiuchi $\mathrm{M}$, et al. The Japanese Society of Hypertension guidelines for the management of hypertension (JSH 2014). Hypertens Res 2014; 37: $253-387$.

14. Kinoshita M, Yokote K, Arai H, Iida M, Ishigaki Y, Ishibashi S, et al. Japan Atherosclerosis Society (JAS) guidelines for prevention of atherosclerotic cardiovascular diseases 2017. J Atheroscler Thromb 2018; 25: 846-984.

15. Japan Diabetes Society. Treatment guide for diabetes 2017, 57th edn [in Japanese]. Tokyo: NANKODO, 2017; 10-11.

16. Committee of the Japan Diabetes Society on the Diagnostic Criteria of Diabetes Mellitus, Seino Y, Nanjo K, Tajima N, Kadowaki T, Kashiwagi A, Araki E, et al. Report of the Committee on the Classification and Diagnostic Criteria of Diabetes Mellitus. J Diabetes Investig 2010; 1: 212-228.

17. Ministry of Internal Affairs and Communications. 2017 Reports from surveillance for trend in usage of information and communication technologies [in Japanese]. https://www.soumu.go.jp/ johotsusintokei/statistics/pdf/HR201700_001.pdf (accessed January $6,2020)$.

18. Ministry of Health, Labour and Welfare. The patients survey, 2011 [in Japanese]. https://www.mhlw.go.jp/index.html (accessed January 6,2020 ). 